\title{
Beyond Reggeization for two- and three-loop QCD amplitudes
}

\author{
Vittorio Del Duca \\ INFN, Laboratori Nazionali di Frascati \\ E-mail: delduca@lnf.infn.it \\ Giulio Falcioni \\ University of Torino and INFN, Sezione di Torino \\ E-mail: falcioni@to.infn.it

\section{Lorenzo Magnea*} \\ University of Torino and INFN, Sezione di Torino \\ E-mail: lorenzo.magnea@unito.it

\section{Leonardo Vernazza} \\ INFN, Sezione di Torino \\ E-mail: vernazza@to.infn.it
}

\begin{abstract}
The high-energy factorization of gauge theory scattering amplitudes in terms of universal impact factors and a Reggeized exchange in the $t$-channel, corresponding to a Regge pole in the angular momentum plane, is know to conflict with the structure of soft anomalous dimensions starting at the two-loop level. We explore the implications of this violation of factorization for two- and three-loop QCD amplitudes: first we propose criteria to organize the amplitudes into factorizing and non-factorizing terms, then we test them by recovering a known result for non-logarithmic terms at two loops. Finally we predict the precise value of the leading non-factorizing energy logarithms at three loops, and we uncover a set of all-order identities constraining infrared finite terms in quark and gluon amplitudes.
\end{abstract}

11th International Symposium on Radiative Corrections (Applications of Quantum Field Theory to Phenomenology) (RADCOR 2013),

22-27 September 2013

Lumley Castle Hotel, Durham, UK

\footnotetext{
* Speaker.
} 


\section{Introduction}

Recent years have seen truly remarkable progress in our understanding of scattering amplitudes in quantum field theory. New techniques have been developed to compute scattering amplitudes of increasing complexity at finite orders in perturbation theory (see for example the recent reviews [1,2]), and new approaches, emerging from special theories, are introducing radically different points of view [3]. Within this fast-developing framework, general properties of amplitudes in special kinematical regimes, notably factorization and universality, continue to provide insights which apply to all orders in perturbations theory, and which prove useful both for testing high-order calculations, and for direct phenomenological applications.

Our goal in this contribution is to examine gauge theory scattering amplitudes using constraints that arise from two different kinematical limits, where two different factorizations, and two different notions of universality, are known to apply. Our main target is the calculation of amplitudes in the high-energy limit, characterized by the fact that the center-of-mass energy $\sqrt{s}$ is much larger than all other kinematic invariants. In this limit, large logarithms of $s$ dominate the perturbative expansion and, at least to some finite logarithmic accuracy, they can be resummed using Regge theory [4]. While the validity of Regge theory rests upon very general principles of quantum field theory, and indeed many related results predate and anticipate subsequent developments in QCD, concrete applications to the perturbative resummation of energy logarithms have been so far confined to leading and next-to-leading logarithms. Our main tool to go beyond existing results for the high-energy limit will be soft-collinear factorization: the statement that leading contributions to scattering amplitudes from virtual particles carrying vanishing energy, or with velocities collinear to the external momenta, can be factorized and thus resummed in exponential form. Soft-collinear factorization is valid to all orders in perturbation theory, but of course applies only to divergent parts of the amplitude.

It is not surprising that information on the high-energy limit can be gained by considering the infrared properties of scattering amplitudes: in the presence of some kinematic cutoff, infrared divergences appear as logarithmic enhancements involving the ratio of the characteristic energy scale of the process with the new scale provided by the cutoff: in this sense, high-energy logarithms can be seen to some extent as a special class of infrared logarithms. This kind of insight has been used to explore the high-energy limit since early days [5, 6, 7]. More recently, a detailed analysis of soft-collinear factorization in the high-energy limit $[8,9]$ showed that the simplest form of Regge factorization, which is based on the approximation of considering only Regge poles as possible singularities in the angular momentum plane, breaks down for infrared divergent contributions at the level of next-to-next-to leading logarithms (NNLL). Conversely, and even more recently, Ref. [10] used constraints derived from Regge factorization to provide evidence for new structures arising at four loops in soft anomalous dimensions.

In this note, we will start from the results of [8,9] and apply them to the explicit calculation of finite order quark and gluon amplitudes in QCD. We begin, in Section 2, by reviewing the basic features of the two factorizations that we shall be employing, and the precise form they take for the case at hand. Then we will show, in Section 3, how finite order constraints can be derived by comparing the two factorization, starting with an illustration at the one-loop level. Our main results [12] are given in Section 4: there, starting at the two-loop level, we show how one can use soft-collinear 
factorization to detect universality-breaking terms at high energy. We organize these terms into remainder functions whose divergent contributions can be determined using infrared information, and we test our ideas at two loops, for non-logarithmic terms, reproducing the results of Ref. [11], where for the first time a violation of Regge factorization was detected. We then proceed to study non-Reggeizing energy logarithms at three loops, and provide explicit predictions for the divergent parts of their coefficients in the case of quark and gluon four-point amplitudes. Such contributions, arising at three loops in non-planar diagrams, are expected to correspond to Regge cuts in the angular momentum plane, and could provide a boundary condition for a more general high-energy resummation, going beyond conventional Reggeization. We conclude by showing how high-energy factorization can, in turn, provide interesting contraints on the finite parts of soft-collinear factorized amplitudes. Our results will be extended to subleading infrared poles and discussed in greater detail in Ref. [13].

\section{A tale of two factorizations}

We begin by describing the framework of high-energy factorization. As in the rest of this note, we will consider the case of four-parton amplitudes in QCD, although the formalism is much more general and in particular can treat multi-parton amplitudes as well. For four partons, in the limit $s \gg|t|$, the amplitude is dominated by color octet exchange in the $t$ channel. It takes on a factorized form, and we will take as master formula for this factorization the expression

$$
\begin{aligned}
\mathscr{M}_{a b}^{[8]}\left(\frac{s}{\mu^{2}}, \frac{t}{\mu^{2}}, \alpha_{s}\right)= & 2 \pi \alpha_{s} H_{a b}^{(0),[8]}\left\{C_{a}\left(\frac{t}{\mu^{2}}, \alpha_{s}\right)\left[A_{+}\left(\frac{s}{t}, \alpha_{s}\right)+\kappa_{a b} A_{-}\left(\frac{s}{t}, \alpha_{s}\right)\right] C_{b}\left(\frac{t}{\mu^{2}}, \alpha_{s}\right)\right. \\
& \left.+\mathscr{R}_{a b}^{[8]}\left(\frac{s}{\mu^{2}}, \frac{t}{\mu^{2}}, \alpha_{s}\right)+\mathscr{O}\left(\begin{array}{l}
t \\
s
\end{array}\right)\right\} .
\end{aligned}
$$

Here the indices $a, b=q, g$ denote the parton species (quark or gluon), and we are not displaying (as in the rest of the paper) the dependence on the IR regulator $\varepsilon=2-d / 2$. The impact factors $C_{a, b}$ are universal functions, depending only on the identity of the particles that scatter by exchanging the $t$-channel color octet. The finite tree-level amplitude $H_{a b}^{(0),[8]}$ contains the $t$-channel propagator pole, while the high-energy logarithms are generated by the Regge factors $A_{ \pm}$, which are given by

$$
A_{ \pm}\left(\frac{s}{t}, \alpha_{s}\right)=\left(\frac{-s}{-t}\right)^{\alpha(t)} \pm\left(\frac{s}{-t}\right)^{\alpha(t)}
$$

where $\alpha(t)$ is the Regge trajectory for octet exchange, which admits a perturbative expansion in powers of $\alpha_{s}$, with IR divergent coefficients. The Regge factors have already been properly (anti)symmetrized with respect to the exchange $s \leftrightarrow u \sim-s$, which also requires the inclusion of the factors $\kappa_{a b}$, compensating for the different symmetry properties of quark and gluon color factors; specifically, $\kappa_{g g}=\kappa_{q g}=0$, while $\kappa_{q q}=\left(4-N_{c}^{2}\right) / N_{c}^{2}$. Finally, in Eq. (2.1) we have allowed for a remainder function $\mathscr{R}_{a b}^{[8]}$, designed to collect all non-factorizing contributions to the amplitude, at leading power in $|t| / s$. High-energy factorization is known to be exact (with vanishing remainder) at leading [14] and next-to-leading [15, 16] logarithmic accuracy (for the real part of the amplitude), while evidence of a non-vanishing, non-logarithmic remainder at two loops was uncovered in Ref. [11]. 
Turning now to soft-collinear factorization, we may start with a general expression generating infrared divergences of arbitrary multi-parton amplitudes [17, 18, 19, 20, 21]. For $n$ partons with momenta $p_{i}$ we may write

$$
\mathscr{M}\left(\frac{p_{i}}{\mu}, \alpha_{s}\right)=\mathscr{Z}\left(\frac{p_{i}}{\mu}, \alpha_{s}\right) \mathscr{H}\left(\frac{p_{i}}{\mu}, \alpha_{s}\right),
$$

where the matrix element $\mathscr{M}$ and the finite hard part $\mathscr{H}$ are vectors in the space of available color tensors, while $\mathscr{Z}$ is an operator in the same space, which generates all infrared poles in dimensional regularization. Multiplicative renormalizability implies that the $\mathscr{Z}$ operator can be written in terms of a soft anomalous dimension matrix $\Gamma$ as

$$
\mathscr{Z}\left(\frac{p_{i}}{\mu}, \alpha_{s}\right)=\mathscr{P} \exp \left[\frac{1}{2} \int_{0}^{\mu^{2}} \frac{d \lambda^{2}}{\lambda^{2}} \Gamma\left(\frac{p_{i}}{\lambda}, \alpha_{s}\left(\lambda^{2}\right)\right)\right] .
$$

The state of the art concerning the soft anomalous dimension matrix $\Gamma$, for massless partons, is expressed by the dipole formula [18, 19, 20, 21]

$$
\Gamma_{\text {dip }}\left(\frac{p_{i}}{\lambda}, \alpha_{s}\left(\lambda^{2}\right)\right)=\frac{1}{4} \widehat{\gamma}_{K}\left(\alpha_{S}\left(\lambda^{2}\right)\right) \sum_{(i, j)} \ln \left(\frac{-s_{i j}}{\lambda^{2}}\right) \mathbf{T}_{i} \cdot \mathbf{T}_{j}-\sum_{i} \gamma_{J_{i}}\left(\alpha_{s}\left(\lambda^{2}\right)\right)
$$

where $\mathbf{T}_{i}$ is a color insertion operator appropriate to parton $i, \widehat{\gamma}_{K}$ is the cusp anomalous dimension $[22,23]$ with the Casimir of the relevant color representation scaled out, and $\gamma_{J_{i}}$ are collinear anomalous dimensions for each external parton. The dipole formula is exact at two loops [24], and at three loops can only receive tightly constrained corrections $[20,25,26]$ whose calculation is under way [27]. Evidence for such corrections at the four-loop level was uncovered in [10].

We now follow Refs. [8, 9], and take the high-energy limit of Eq. (2.3), using Eq. (2.5). For four-point amplitudes, to leading power in $|t| / s$, but to all logarithmic accuracies, the infrared operator $\mathscr{Z}$ factorizes as

$$
\mathscr{Z}\left(\frac{p_{i}}{\mu}, \alpha_{s}\right)=\mathscr{Z}_{\mathbf{1}, \mathbf{R}}\left(\frac{t}{\mu^{2}}, \alpha_{s}\right) \exp \left(-\mathrm{i} \frac{\pi}{2} K\left(\alpha_{s}\right) \mathscr{C}_{\text {tot }}\right) \widetilde{\mathscr{Z}}\left(\frac{s}{t}, \alpha_{s}\right)+\mathscr{O}\left(\frac{t}{s}\right) .
$$

The crucial ingredient of Eq. (2.6), which retains a matrix structure in color space, and generates all energy logarithms, is the factor

$$
\widetilde{\mathscr{Z}}\left(\frac{s}{t}, \alpha_{s}\right)=\exp \left\{K\left(\alpha_{s}\right)\left[\log \left(\frac{s}{-t}\right) \mathbf{T}_{t}^{2}+\mathrm{i} \pi \mathbf{T}_{s}^{2}\right]\right\},
$$

where we have introduced the 'Mandelstam' combinations of color operators $\mathbf{T}_{t}=\mathbf{T}_{1}+\mathbf{T}_{3}$ and $\mathbf{T}_{s}=\mathbf{T}_{1}+\mathbf{T}_{2}$ for the scattering process $1+2 \rightarrow 3+4$, and we defined

$$
K\left(\alpha_{s}\right)=-\frac{1}{4} \int_{0}^{\mu^{2}} \frac{d \lambda^{2}}{\lambda^{2}} \hat{\gamma}_{K}\left(\alpha_{s}\left(\lambda^{2}\right)\right) .
$$

Furthermore, in Eq. (2.6) we have isolated a divergent phase, defining the Casimir eigenvalue $\mathscr{C}_{\text {tot }}$ as the sum of the four Casimirs associated to the four external partons. The remaining factor, $\mathscr{Z}_{\mathbf{1}, \mathbf{R}}$ is then a real, color singlet, energy-independent exponential of the form

$$
\mathscr{Z}_{\mathbf{1}, \mathbf{R}}\left(\frac{t}{\mu^{2}}, \alpha_{s}\right)=\exp \left\{\frac{1}{2}\left[K\left(\alpha_{s}\right) \log \left(\frac{-t}{\mu^{2}}\right)+D\left(\alpha_{s}\right)\right] \mathscr{C}_{\text {tot }}+\sum_{i=1}^{4} B_{i}\left(\alpha_{s}\right)\right\},
$$


where the functions $D\left(\alpha_{s}\right)$ and $B_{i}\left(\alpha_{s}\right)$ are scale integrals similar to Eq. (2.8), and given explicitly in Ref. [8]. The most relevant feature of Eq. (2.9) for our present purposes is that it can be written as a product of factors unambiguously associated with each external parton, as

$$
\mathscr{Z}_{\mathbf{1}, \mathbf{R}}\left(\frac{t}{\mu^{2}}, \alpha_{s}\right)=\prod_{i=1}^{4} \mathscr{Z}_{\mathbf{1}, \mathbf{R}}^{(i)}\left(\frac{t}{\mu^{2}}, \alpha_{s}\right) .
$$

Such an expression strongly suggests that the factors $\mathscr{Z}_{\mathbf{1}, \mathbf{R}}^{(i)}$ should be related to the (divergent contributions to) the impact factors $C_{a}$. As we will see in the next sections, this is indeed the case.

\section{Perturbative expansions}

Our task is now in principle straightforward: we must expand the all-order factorized expressions given in the previous section in powers of the coupling and of the energy logarithm; comparing the resulting perturbative coefficients will make explicit the constraints that each factorization implies for the other one, and will give explicit results for the violations of high-energy factorization at NNLL that were described in Refs. [8,9]. For example, we expand each color component $\mathscr{M}^{[j]}$ of the full matrix elements as

$$
\mathscr{M}^{[j]}\left(\frac{s}{\mu^{2}}, \frac{t}{\mu^{2}}, \alpha_{s}\right)=4 \pi \alpha_{s} \sum_{n=0}^{\infty} \sum_{i=0}^{n}\left(\frac{\alpha_{s}}{\pi}\right)^{n} \ln ^{i}\left(\frac{s}{-t}\right) M^{(n), i,[j]}\left(\frac{t}{\mu^{2}}\right),
$$

and similarly for the remainder functions $\mathscr{R}_{a b}^{[8]}$, where however we know that leading contributions must arise at $\mathscr{O}\left(\alpha_{s}^{2}\right)$, and be next-to-next-to leading in the energy logarithm. All functions that do not depend on the energy such as impact factors and the singlet operator $\mathscr{Z}_{\mathbf{1}, \mathbf{R}}$ are simply expanded in powers of $\alpha_{s} / \pi$.

Let us illustrate the procedure at the one loop level. Soft-collinear factorization at this level gives the expressions

$$
\begin{aligned}
& M^{(1), 0}=\left[Z_{1, \mathbf{R}}^{(1)}+i \pi K^{(1)}\left(\mathbf{T}_{s}^{2}-\frac{1}{2} \mathscr{C}_{\text {tot }}\right)\right] H^{(0)}+H^{(1), 0}, \\
& M^{(1), 1}=K^{(1)} \mathbf{T}_{t}^{2} H^{(0)}+H^{(1), 1},
\end{aligned}
$$

which are still vector equations in color space, whereas, expanding Eq. (2.1), we readily find that the color octet components of the one-loop matrix elements can be expressed as

$$
\begin{aligned}
& M_{a b}^{(1), 0,[8]}=\left[C_{a}^{(1)}+C_{b}^{(1)}-i \frac{\pi}{2}\left(1+\kappa_{a b}\right) \alpha^{(1)}\right] H_{a b}^{(0),[8]}, \\
& M_{a b}^{(1), 1,[8]}=\alpha^{(1)} H_{a b}^{(0),[8]} .
\end{aligned}
$$

Comparing the two results for the octet component at LL level, we readily verify that the one-loop Regge trajectory must be given by

$$
\alpha^{(1)}=\frac{K^{(1)}\left(\mathbf{T}_{t}^{2} H^{(0)}\right)^{[8]}}{H^{(0),[8]}}+\frac{H^{(1), 1,[8]}}{H^{(0),[8]}}=C_{A} K^{(1)}+\mathscr{O}(\varepsilon),
$$

as predicted in $[8,9]$. Note that, to get the second equality, we made use of the fact that the tree-level matrix element is pure color octet at leading power in $|t| / s$, and we used the fact that $H^{(1), 1,[8]}=$ 
$\mathscr{O}(\varepsilon)$. On the other hand, the reality of the Regge trajectory requires that $\operatorname{Im}\left(H^{(1), 1,[8]}\right)=0$, which is easily verified. More interesting constraints arise when looking at the NLL terms in Eqns. (3.2) and (3.3). Concentrating on imaginary parts, one finds that

$$
\operatorname{Im}\left[H^{(1), 0,[8]}\right]=-\frac{\pi}{2}(1+\kappa) \operatorname{Re}\left[H^{(1), 1,[8]}\right]+\frac{\pi}{2} K^{(1)}\left(\left[\mathscr{C}_{\mathrm{tot}}-2 \mathbf{T}_{s}^{2}-(1+\kappa) \mathbf{T}_{t}^{2}\right] H^{(0)}\right)^{[8]}
$$

Now, soft-collinear factorization requires $H^{(1), 0,[8]}$ to be finite, while $K^{(1)}$ is a pure pole. Consistency requires the vanishing of the matrix element

$$
\left[\mathscr{C}_{\mathrm{tot}}-2 \mathbf{T}_{s}^{2}-(1+\kappa) \mathbf{T}_{t}^{2}\right]_{[8],[8]}=0,
$$

for any combination of representations that can participate in the scattering process. Using, for example, the explicit results for the color bases constructed in [28], one can check that this identity is in fact verified. Coming finally to the real part of the NLL matrix elements, one finds from quarkquark scattering and gluon-gluon scattering respectively that the corresponding impact factors must be given by

$$
C_{a}^{(1)}=\frac{1}{2} Z_{1, \mathbf{R}, a}^{(1)}+\frac{1}{2} \widehat{H}_{a a}^{(1), 0,[8]},
$$

where we defined $\widehat{H}^{(1), 0,[8]} \equiv H^{(1), 0,[8]} / H^{0,[8]}$. Quark-gluon scattering is then completely determined, if universality has to hold. This is easily verified to be true for divergent terms, using the explicit expressions for $\mathscr{Z}_{\mathbf{1}, \mathbf{R}}^{(i)}$. For finite contributions one needs

$$
\operatorname{Re}\left(\widehat{H}_{q g}^{(1), 0,[8]}\right)=\frac{1}{2}\left[\operatorname{Re}\left(\widehat{H}_{g g}^{(1), 0,[8]}\right)+\operatorname{Re}\left(\widehat{H}_{q q}^{(1), 0,[8]}\right)\right]
$$

which can also be verified using the one-loop results derived in [29].

\section{Results at two loops and beyond}

The results presented in Section 3 are of course not new, and serve mostly to illustrate the method we use. Things get more interesting starting at two loops. At this level, the LL results simply confirm the exponentiation implicit in the factorized expression (2.1). On the other hand the real part of the single-logarithmic NLL matrix elements provide an expression for the two-loop Regge trajectory,

$$
\alpha^{(2)}=C_{A} K^{(2)}+\operatorname{Re}\left[\widehat{H}_{a b}^{(2), 1,[8]}\right]+\mathscr{O}(\varepsilon)
$$

which again corresponds both to expectations and to known results. Yet more interesting are the results for impact factors obtained by fitting the real parts of the NNLL matrix elements. Indeed one finds

$$
\begin{gathered}
C_{a}^{(2)}=\frac{1}{2} Z_{1, \mathbf{R}, a a}^{(2)}-\frac{1}{8}\left(Z_{1, \mathbf{R}, a a}^{(1)}\right)^{2}+\frac{1}{4} Z_{1, \mathbf{R}, a a}^{(1)} \operatorname{Re}\left[\widehat{H}_{a a}^{(1), 0,[8]}\right]-\frac{1}{4} R_{a a}^{(2), 0,[8]} \\
-\frac{\pi^{2}\left(K^{(1)}\right)^{2}}{4}\left\{\left[\left(\mathbf{T}_{s, a a}^{2}\right)^{2}\right]_{[8],[8]}-\mathscr{C}_{\mathrm{tot}, a a}\left[\mathbf{T}_{s, a a}^{2}\right]_{[8],[8]}+\frac{1}{4} \mathscr{C}_{\mathrm{tot}, a a}^{2}-\frac{\left(1+\kappa_{a a}\right) C_{A}^{2}}{2}\right\}+\mathscr{O}\left(\varepsilon^{0}\right) .
\end{gathered}
$$

If high-energy factorization were to be exact at this order, the remainders $R_{a a}^{(2), 0,[8]}$ would vanish. One sees immediately however that this cannot work: indeed, while the first line of Eq. (4.2) would 
have the proper degree of universality, the second line involves both mixing of color components through the $s$-channel color operator $\mathbf{T}_{s}$, as well as process-dependent terms. We conclude that Regge factorization as embodied in Eq. (2.1) must break down, and Eq. (4.2) provides us with the tools to isolate the factorization-breaking terms. We can define universal impact factors using the first line of Eq. (4.2), as

$$
\widetilde{C}_{a}^{(2)}=\frac{1}{2} Z_{1, \mathbf{R}, a a}^{(2)}-\frac{1}{8}\left(Z_{1, \mathbf{R}, a a}^{(1)}\right)^{2}+\frac{1}{4} Z_{1, \mathbf{R}, a a}^{(1)} \operatorname{Re}\left[\widehat{H}_{a a}^{(1), 0,[8]}\right]+\mathscr{O}\left(\varepsilon^{0}\right),
$$

while the second line naturally defines non-factorizing remainders $\widetilde{R}_{a b}^{(2), 0,[8]}$. We can check the consistency of our approach at the two-loop level: first we can compute the newly defined remainders for quark and gluon amplitudes by using the explicit color bases of Ref. [28], with the results

$$
\widetilde{R}_{q q}^{(2), 0,[8]}=\frac{\pi^{2}}{4 \varepsilon^{2}}\left(1-\frac{3}{N_{c}^{2}}\right), \quad \widetilde{R}_{g g}^{(2), 0,[8]}=-\frac{3 \pi^{2}}{2 \varepsilon^{2}}, \quad \widetilde{R}_{q g}^{(2), 0,[8]}=-\frac{\pi^{2}}{4 \varepsilon^{2}} .
$$

Next, we can construct a function measuring the discrepancy between the predictions of Regge factorization for the quark-gluon amplitude, which are based on universality, and the actual matrix elements. We find

$$
\begin{aligned}
\Delta_{(2), 0,[8]} & \equiv \frac{M_{q g}^{(2), 0}}{H_{q g}^{(0),[8]}}-\left[C_{q}^{(2)}+C_{g}^{(2)}+C_{q}^{(1)} C_{g}^{(1)}-\frac{\pi^{2}}{4}(1+\kappa)\left(\alpha^{(1)}\right)^{2}\right] \\
& =\frac{1}{2}\left[\widetilde{R}_{q g}^{(2), 0,[8]}-\frac{1}{2}\left(\widetilde{R}_{q q}^{(2), 0,[8]}+\widetilde{R}_{g g}^{(2), 0,[8]}\right)\right]=\frac{\pi^{2}}{\varepsilon^{2}} \frac{3}{16}\left(\frac{N_{c}^{2}+1}{N_{c}^{2}}\right),
\end{aligned}
$$

which precisely reproduces the result of Ref. [11].

Clearly, soft-collinear factorization can be used to identify precisely, order by order in perturbation theory, the non-universal terms that break Regge factorization. Moving on to the threeloop level, one finds, as expected, that the breaking of universality occurs at the level of singlelogarithmic terms. Indeed, if one attempts to find an expression for the three-loop Regge trajectory using soft-collinear ingredients one finds

$$
\begin{aligned}
\alpha^{(3)}= & C_{A} K^{(3)}+\frac{\pi^{2}\left(K^{(1)}\right)^{3}}{2}\left[\mathscr{C}_{\mathrm{tot}, a b} N_{c}\left(\mathbf{T}_{s, a b}^{2}\right)_{[8],[8]}-\frac{\mathscr{C}_{\mathrm{tot}, a b}^{2} N_{c}}{4}+\frac{1+\kappa_{a b}}{2} N_{c}^{3}\right. \\
& \left.-\frac{1}{3} \sum_{n}\left(2 N_{c}+\mathscr{C}_{[n]}\right)\left|\left(\mathbf{T}_{s, a b}^{2}\right)_{[8], n}\right|^{2}\right]-\frac{1}{2} R_{a b}^{(3), 1,[8]}+\mathscr{O}\left(\varepsilon^{-2}\right),
\end{aligned}
$$

where lower-order poles can also be determined [13] but are omitted here for brevity. Once again, the first term in Eq. (4.6) is universal and has the expected form, while the second term involves both color mixing and process-dependent contributions. Following our general strategy, we define

$$
\widetilde{\alpha}^{(3)}=K^{(3)} N_{c}+\mathscr{O}\left(\varepsilon^{0}\right) .
$$

We can now determine the three-loop, single logarithmic remainders, by computing the nonuniversal contributions to Eq. (4.6) in the color bases of Ref. [28]. The results for quark and gluon amplitudes are

$$
\widetilde{R}_{q q}^{(3), 1,[8]}=\frac{\pi^{2}}{\varepsilon^{3}} \frac{2 N_{c}^{2}-5}{12 N_{c}}, \quad \widetilde{R}_{g g}^{(3), 1,[8]}=-\frac{\pi^{2}}{\varepsilon^{3}} \frac{2}{3} N_{c}, \quad \widetilde{R}_{q g}^{(3), 1,[8]}=-\frac{\pi^{2}}{\varepsilon^{3}} \frac{N_{c}}{24} .
$$


Together with Eq. (2.1), Eq. (4.8) constitutes an explicit prediction for the high-energy limit of quark and gluon amplitudes at three loops.

We conclude by noting that, just as soft-collinear factorization provides important information in the high-energy limit, one may also use Regge factorization to extract constraints on the finite parts of the amplitudes, which are not in principle controlled by infrared physics. An example of this was discussed at the one-loop level in Section 3, most notably in Eqns. (3.5) and (3.6): there, the reality of the Regge trajectory and impact factors was reflected in properties of the one-loop hard imaginary parts. It turns out that it possible to generalize these constraints to all orders in perturbation theory, using extensions of the color identity given in Eq. (3.6). For the LL and NLL hard-scattering coefficients we find [13]

$$
\begin{aligned}
\operatorname{Im}\left(\widehat{H}^{(n), n,[8]}\right) & =0 \\
\operatorname{Re}\left(\widehat{H}^{(n), n,[8]}\right) & =\frac{1}{n !}\left(\widehat{H}^{(1), 1,[8]}\right)^{n}=O\left(\varepsilon^{n}\right) \\
\operatorname{Im}\left(\widehat{H}^{(n), n-1,[8]}\right) & =-\pi \frac{1+\kappa}{2}\left(n \widehat{H}^{(n), n,[8]}\right)=O\left(\varepsilon^{n}\right), \\
\operatorname{Re}\left(\widehat{H}^{(n), n-1,[8]}\right) & =\operatorname{Re}\left(\widehat{H}^{(2), 1}\right) \widehat{H}^{(n-2), n-2}+(2-n) \operatorname{Re}\left(\widehat{H}^{(1), 0,[8]}\right) \widehat{H}^{(n-1), n-1}=\mathscr{O}\left(\varepsilon^{n-2}\right) .
\end{aligned}
$$

In essence, Eq. (4.9) reinforces the idea that high-energy logarithms are in fact infrared in nature: indeed, leading and next-to-leading logarithmic contributions to hard scattering coefficients are forced to vanish with increasing powers of the regulator $\varepsilon$. This means that infrared-finite highenergy logarithms must come from the interference of soft and collinear functions with lower-order contributions subleading in $\varepsilon$. These constraints, discussed in greater detail in [13], are the subject of ongoing investigations.

\section{Acknowledgements}

This work was supported by MIUR (Italy), under contract 2010YJ2NYW_006; by the University of Torino and by the Compagnia di San Paolo under contract ORTO11TPXK; by the Research Executive Agency (REA) of the European Union, through the Initial Training Network LHCPhenoNet under contract PITN-GA-2010-264564, and by the ERC grant 291377 "LHCtheory: Theoretical predictions and analyses of LHC physics: advancing the precision frontier".

\section{References}

[1] H. Elvang and Y. -t. Huang, arXiv:1308.1697 [hep-th].

[2] L. J. Dixon, arXiv:1310.5353 [hep-ph].

[3] N. Arkani-Hamed, J. L. Bourjaily, F. Cachazo, A. B. Goncharov, A. Postnikov and J. Trnka, arXiv:1212.5605 [hep-th].

[4] P. D. B. Collins, “An Introduction to Regge Theory and High-Energy Physics”, Cambridge 1977, 445 p. 
[5] G. P. Korchemsky, Phys. Lett. B325 (1994) 459, hep-ph/ 9311294.

[6] I. A. Korchemskaya, G. P. Korchemsky, Nucl. Phys. B437 (1995) 127, hep-ph/ 9409446.

[7] I. A. Korchemskaya, G. P. Korchemsky, Phys. Lett. B387 (1996) 346, hep-ph/ 9607229.

[8] V. Del Duca, C. Duhr, E. Gardi, L. Magnea and C. D. White, JHEP 1112 (2011) 021, arXiv:1109.3581 [hep-ph].

[9] V. Del Duca, C. Duhr, E. Gardi, L. Magnea and C. D. White, Phys. Rev. D 85 (2012) 071104, arXiv:1108.5947 [hep-ph].

[10] S. Caron-Huot, arXiv:1309.6521 [hep-th].

[11] V. Del Duca and E. W. N. Glover, JHEP 0110 (2001) 035, hep-ph / 0109028.

[12] V. Del Duca, G. Falcioni, L. Magnea and L. Vernazza, arXiv:1311.0304 [hep-ph] .

[13] V. Del Duca, G. Falcioni, L. Magnea and L. Vernazza, in preparation.

[14] I. I. Balitsky, L. N. Lipatov and V. S. Fadin, In *Leningrad 1979, Proceedings, Physics Of Elementary Particles*, Leningrad 1979, 109-149.

[15] V. S. Fadin and L. N. Lipatov, Nucl. Phys. B 406 (1993) 259.

[16] V. S. Fadin, R. Fiore, M. G. Kozlov and A. V. Reznichenko, Phys. Lett. B 639 (2006) 74, hep-ph/0602006.

[17] L. J. Dixon, L. Magnea and G. F. Sterman, JHEP 0808 (2008) 022, arXiv : 0805.3515 [hep-ph] .

[18] T. Becher and M. Neubert, Phys. Rev. Lett. 102 (2009) 162001, arXiv:0901.0722 [hep-ph] .

[19] E. Gardi and L. Magnea, JHEP 0903 (2009) 079, arXiv: 0901.1091 [hep-ph] .

[20] T. Becher and M. Neubert, JHEP 0906 (2009) 081, arXiv: 0903.1126 [hep-ph] .

[21] E. Gardi and L. Magnea, Nuovo Cim. C 32N5-6 (2009) 137, arXiv: 0908.3273 [hep-ph] .

[22] G. P. Korchemsky and A. V. Radyushkin, Phys. Lett. B 171 (1986) 459.

[23] G. P. Korchemsky and A. V. Radyushkin, Nucl. Phys. B 283 (1987) 342.

[24] S. M. Aybat, L. J. Dixon and G. F. Sterman, Phys. Rev. D 74 (2006) 074004, hep-ph $/ 0607309$.

[25] L. J. Dixon, E. Gardi and L. Magnea, JHEP 1002 (2010) 081, arXiv: 0910.3653 [hep-ph].

[26] V. Ahrens, M. Neubert and L. Vernazza, JHEP 1209 (2012) 138, arXiv:1208. 4847 [hep-ph] .

[27] E. Gardi, arXiv:1310.5268 [hep-ph].

[28] M. Beneke, P. Falgari and C. Schwinn, Nucl. Phys. B 828 (2010) 69, arXiv: 0907.1443 [hep-ph] .

[29] Z. Kunszt, A. Signer and Z. Trocsanyi, Nucl. Phys. B 411 (1994) 397, hep-ph/ 9305239. 